\title{
A compact low insertion loss bandpass filter based on meandered self-coupled ring resonator
}

\author{
Ching-Chien Cheng ${ }^{1, *}$, Kong-Xin Cheng ${ }^{1}$, Huang-Kuang Kung ${ }^{1}$, Chin-Yu Wang ${ }^{1}$, and Yng-Huey Jeng ${ }^{2}$ \\ ${ }^{1}$ Institute of Mechatronics Engineering, Cheng Shiu University, Kaohsiung City, Taiwan \\ ${ }^{2}$ Department of Electronic Engineering, National Formosa University, Yunlin, Taiwan
}

\begin{abstract}
The traditional structure of dual mode bandpass filter is formed by a circular or rectangular resonator, which causes excessive circuit areas to be occupied. A $5.2 \mathrm{GHz}$ bandpass filter, based on the triple-branch self-coupled ring resonator, is designed to achieve most essential features such as low insertion loss, compact size, and wide stopband. The coupling of each coupled branch introduces the even- and odd-mode perturbation, which produce sharp bandpass and wide stopband responses due to the generation of band-edge transmission zeros. The simulation and measurement in this paper are respectively verified by using IE3D electromagnetic simulator and Agilent's HP8722C network analyzer. Experimental results show that the filter has $19.2 \%$ bandwidth centered at $5.2 \mathrm{GHz}, 1.2 \mathrm{~dB}$ insertion loss, $25 \mathrm{~dB}$ stopband rejection from 6 to $9 \mathrm{GHz}$. Moreover, the circuit size in this paper has been down to $9 \times 6 \mathrm{~mm}^{2}$ and can be applied to communication of microwave applications.
\end{abstract}

\section{Introduction}

The component of passive circuit, such as the filter occupying the largest percentage of communication system structure, is more important to preserve high performance and reduce the electronic circuit size. Many researches of dual-mode bandpass filter have been proposed in papers [1-9].

The bandpass filters based on the dual-mode ring resonator was first analyzed by Wolff [1]. Some papers proposed dual mode ring resonator bandpass filters by using step-impedance or ring resonators with different perturbation schemes [2-4]. The perturbation element is a plus to or cut from the resonator, and it is also viewed as capacitive or inductive [5]. One of the methods is the investigation of designing a compact, low insertion loss and sharp rejection by cascaded dual mode ring resonator [6]. Furthermore, dual-mode microstrip filters are highly attractive for radio frequency systems because their sizes are compact and easy to fabricate [7]. By adding open stubs symmetrically on ring resonator, the dual mode filter is realized [8].

However, there is a novel distributed perturbation by self-coupled segments of the ring resonator, which brings the easier implementation scheme [9]. In this paper, the ring resonator is based on the theory of a selfcoupled, simple bandpass filter with low insertion loss and the compact size designed, which showed the easy structure and narrow bandwidth with two transmission zeros near the passband. This filter of $5.2 \mathrm{GHz}$ band is designed. The measurement results show good consensus with simulation.

\section{Filter design}

The traditional structure of dual mode bandpass filter is shown in Fig. 1. Generally, the filter structure is formed by a circular or rectangular resonator, which causes excessive circuit areas to be occupied. To make up for the disadvantage, the ring resonator was bended in this study to achieve the goal of saving the filter size.

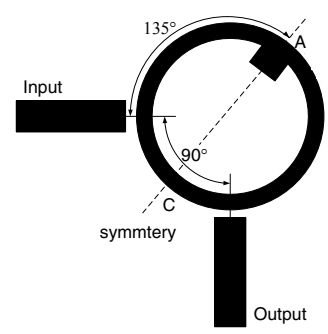

Fig. 1. Structure of traditional dual mode bandpass filter.

\subsection{Schematic of proposed bandpass filter}

Unlike those studies in the past using the perturbation approach, the ring resonator with coupling method is applied to cause the even and odd mode to be separated, and to achieve resonance effects. Thus, the filter offers an advantage of a simpler circuit layout and is easier to be implemented. The schematic types of the proposed self-coupled bandpass filter with input and output ports are shown in Fig. 2. The proposed bandpass filter is composed of self-coupled ring resonator with parallel-

Corresponding author: chching@kmvs.km.edu.tw 
coupled input and output networks. The self-coupled ring includes a central coupled branch and two other coupled branches, connected at both ends of the central coupled branch. An intuitive way to understand this selfcoupled ring resonator is the meandering of the conventional circular ring resonator such that the ring size can be considerably miniaturized. Different from the lumped perturbation of the conventional ring resonator, the distributed coupling of the proposed ring resonator provides the mode perturbation between even and odd modes.

\subsection{Resonance conditions}

In Fig. 2, the layout discontinuity effect of both coupled sections are neglected, with the structure of the ring resonator symmetric to line $\overline{A B}$, and the even-odd mode theory applied to analyze the resonance mode characteristics. Fig. 3(a) and (b) show that the equivalent even-mode, odd-mode circuits, the even-mode and oddmode resonance conditions can be derived from equation (1)-(6) and ignore the coupling support effect on both sides of the bent section. Two coupled sections at ends have the same parameters: the electrical length of $\theta_{1}$, the coupling coefficient of $c_{1}$, and the characteristic impedance $Z_{1}$. The central coupled section has the electrical length of $\theta_{2}$, the coupling coefficient of $c_{2}$, and the characteristic impedance of $Z_{2}$.

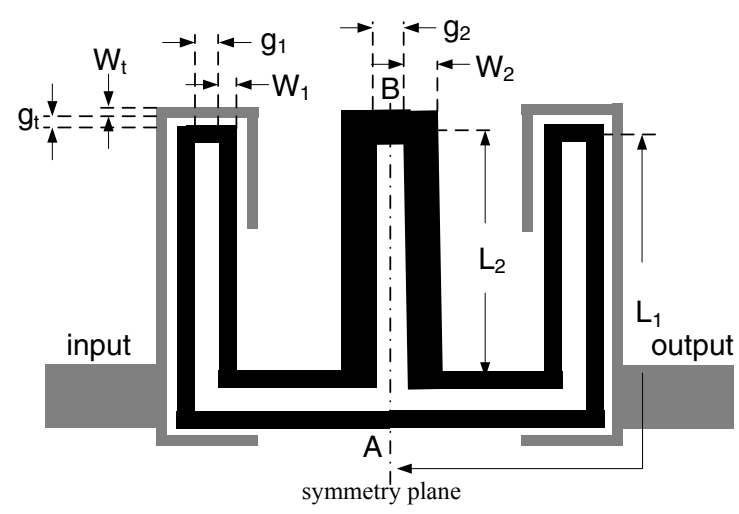

Fig. 2. Structure of proposed coupled-ring bandpass filter.

The equation derived is shown as below. The equivalent even circuit of dual-mode ring resonator is shown in Fig. 3 (a). By calculating the input impedance of the even mode, the even mode resonates when $Z_{B e}=\infty$. On the other hand, the input admittance of the even mode $Y_{B e}=0$.

$$
Y_{B e}=j Y_{2 e} \frac{Z_{2 e}-\frac{1}{2}\left(Z_{1 o} \tan \frac{\theta_{1 e}}{2}-Z_{1 e} \cot \frac{\theta_{1 e}}{2}\right) \tan \theta_{2 e}}{\frac{1}{2}\left(Z_{1 o} \tan \frac{\theta_{1 e}}{2}-Z_{1 e} \cot \frac{\theta_{1 e}}{2}\right)+Z_{2 e} \tan \theta_{2 e}}=0
$$

Then

$$
Z_{2 e}-\frac{1}{2}\left(Z_{1 o} \tan \frac{\theta_{1 e}}{2}-Z_{1 e} \cot \frac{\theta_{1 e}}{2}\right) \tan \theta_{2 e}=0
$$

The even mode resonance condition becomes

$$
\tan \theta_{2 e}=\frac{2 \sqrt{\frac{1+C_{2}}{1-C_{2}}}}{Z\left(\sqrt{\frac{1-C_{1}}{1+C_{1}}} \tan \frac{\theta_{1 e}}{2}-\sqrt{\frac{1+C_{1}}{1-C_{1}}} \cot \frac{\theta_{1 e}}{2}\right)}
$$

Where

$C_{1}=\frac{Z_{1 e}-Z_{1 o}}{Z_{1 e}+Z_{1 o}}, C_{2}=\frac{Z_{2 e}-Z_{2 o}}{Z_{2 e}+Z_{2 o}}, Z_{1}=\sqrt{Z_{1 e} Z_{1 o}}, Z_{2}=\sqrt{Z_{2 e} Z_{2 o}}$ $Z=\frac{Z_{1}}{Z_{2}}$

$$
\frac{\theta_{2 e}}{\theta_{2}}=\frac{f_{r e}}{f_{\text {norm }}}=\frac{\theta_{1 e}}{\theta_{1}} ; \theta_{1}+\theta_{2}=\pi
$$

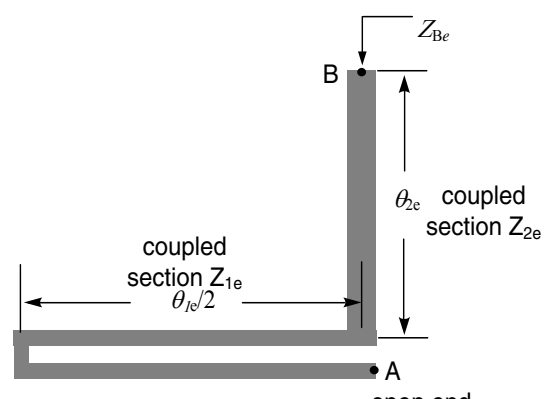

(a) even mode open end

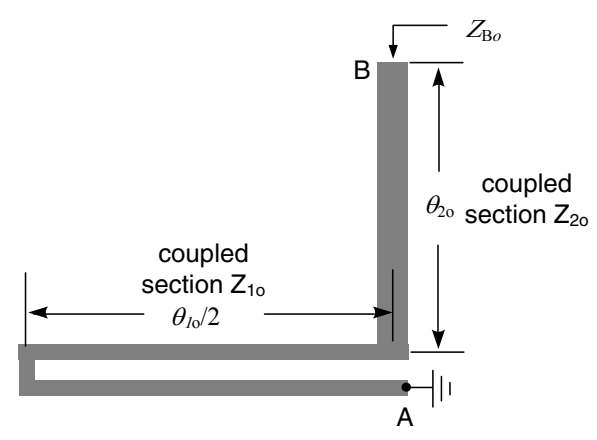

(b) odd mode

Fig. 3. Equivalent even and odd-mode circuit of dual-mode ring resonator.

Similarly, The equivalent odd circuit of dual-mode ring resonator is shown in Fig. 3 (b), and the input impedance vanishes when the odd mode resonates,

$$
Z_{B o}=j Z_{2 o} \frac{\left(Z_{1 e} \cot \frac{\theta_{1 o}}{2}-Z_{1 o} \tan \frac{\theta_{1 o}}{2}\right) \tan \theta_{2 o}+\frac{2 Z_{1 e} Z_{1 o}}{Z_{2 o}}}{\left(Z_{1 e} \cot \frac{\theta_{1 o}}{2}-Z_{1 o} \tan \frac{\theta_{1 o}}{2}\right)-\frac{2 Z_{1 e} Z_{1 o}}{Z_{2 o}} \tan \theta_{2 o}}=0
$$

Then

$$
\left(Z_{1 e} \cot \frac{\theta_{1 o}}{2}-Z_{1 o} \tan \frac{\theta_{1 o}}{2}\right) \tan \theta_{2 o}+\frac{2 Z_{1 e} Z_{1 o}}{Z_{2 o}}=0
$$

The odd mode resonance condition is obtained as 


$$
\tan \theta_{2 o}=\frac{2 Z \sqrt{\frac{1+C_{2}}{1-C_{2}}}}{\left(\sqrt{\frac{1-C_{1}}{1+C_{1}}} \tan \frac{\theta_{1 o}}{2}-\sqrt{\frac{1+C_{1}}{1-C_{1}}} \cot \frac{\theta_{1 o}}{2}\right)}
$$

Where

$C_{1}=\frac{Z_{1 e}-Z_{1 o}}{Z_{1 e}+Z_{1 o}}, C_{2}=\frac{Z_{2 e}-Z_{2 o}}{Z_{2 e}+Z_{2 o}}, Z_{1}=\sqrt{Z_{1 e} Z_{1 o}}, Z_{2}=\sqrt{Z_{2 e} Z_{2 o}}$

$Z=\frac{Z_{1}}{Z_{2}}$

$\frac{\theta_{2 o}}{\theta_{2}}=\frac{f_{r o}}{f_{\text {norm }}}=\frac{\theta_{1 o}}{\theta_{1}} ; \theta_{1}+\theta_{2}=\pi$

Under the even-odd mode resonant frequency $f_{r e}$ and $f_{r o}$, the $\theta_{1 e}, \theta_{2 e}, \theta_{1 o}$ and $\theta_{2 o}$ are the electrical lengths corresponding to the physical lengths $\ell_{1}$ and $\ell_{2}$ respectively. Thus, the resonance frequency condition is obtained as $f_{r}=\sqrt{f_{r e} \cdot f_{r o}}$.

Since the complicated form of equation (1)-(6) is difficult to obtain the analytic solution, we use the graphic expression for analyzing the effect of the evenmode and odd-mode resonant frequencies with the impedance ratio and coupling coefficients.

\subsubsection{Impedance ratio effect $Z$}

A case about $c_{1}=0.2$ and $c_{2}=0.2$ is plotted in Fig. 4 and it shows the dependence of mode resonant frequencies with respect to the impedance ratio of $\mathrm{Z}$ with several characteristics as below.

1) When impedance ratio $Z=1$, the even- and oddmode resonant frequency are not separated.

2) When impedance ratio $Z<1$, the odd-mode resonant is lower than the even-mode and has the inductive perturbation feature.

3) When impedance ratio $\mathrm{Z}>1$, the odd-mode resonant frequency is higher than the even-mode and the type is in a capacitive perturbation state.

4) The geometric average resonant frequency, $f_{r, S C R}=\sqrt{f_{r e} f_{r o}}$ is apathetical to Z.

Therefore, these arguments are determined to support the operating band and bandwidth of the filter in this paper.

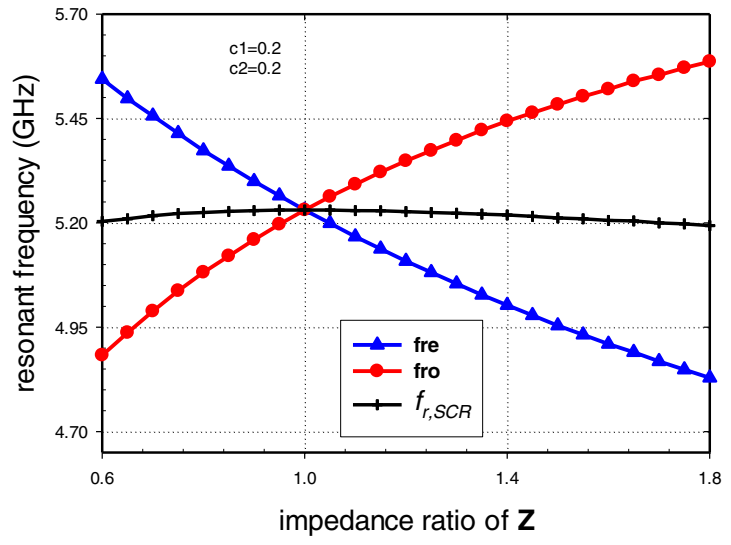

Fig. 4. Resonant frequencies with respect to the impedance ratio of $Z$.

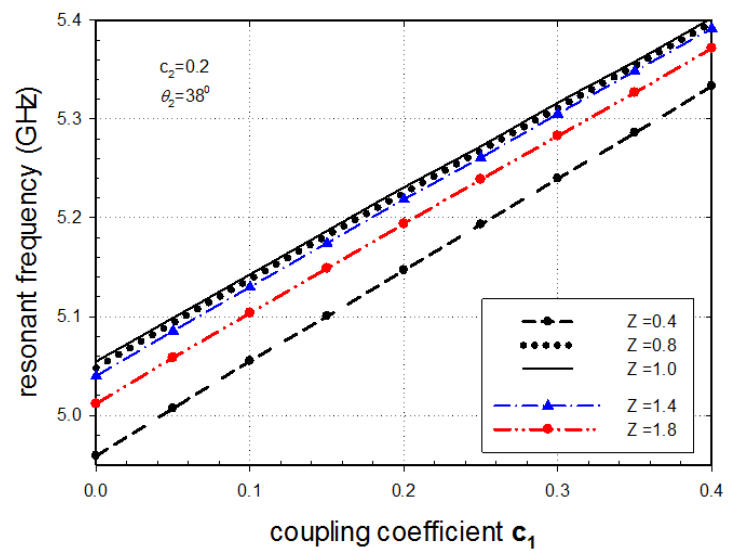

(a) coupling coefficient $\mathrm{C}_{1}$

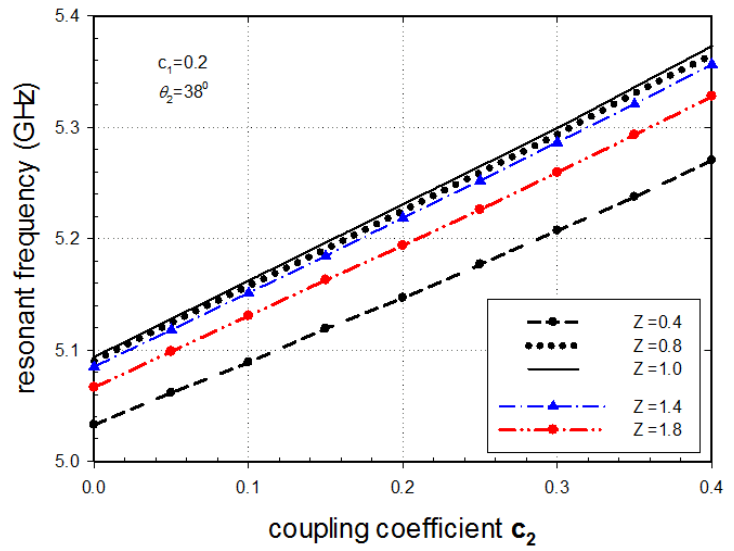

(b) coupling coefficient $\mathrm{C}_{2}$

Fig. 5. Resonant frequencies with respect to the coupling coefficient.

\subsubsection{Characteristics of Coupling Coefficient}

Fig. 5 (a) and (b) are the relative resonant frequency between coupling coefficient $\mathrm{C}_{1}$ and $\mathrm{C}_{2}$ for different impedance ratios under the $\theta_{2}=38^{\circ}$. There are several characteristics as below.

1) When the coupling coefficient increases, the resonant frequency will increase at any impedance ratio. 
2) When $Z<1$, the resonant frequency will increase following the incremental impedance ratio in the any coupling coefficient.

3) When $Z>1$, the the resonant frequency will decrease following the incremental impedance ratio in the any coupling coefficient.

4) When $Z=1$, it shows the highest resonant frequency.

\subsection{Transmission zeros analysis}

The main advantage of the ring resonator filter is generating two transmission zeros near the passband. Because there are two transmission paths between the input and output ports, therefore when the current of the two paths is offset each other at the output, the transmission zeroes on both sides of the passband are generated. In this way, Y-parameter matrix method (Admittance Matrix Method) is used to facilitate the analysis of transmission zero generation.

Ignoring the discontinuous effect of the circuit branch bending, as shown in Fig. 6, the entire resonator can be disassembled into a parallel coupling line with electrical length $\theta_{1} / 2$, impedance $Z_{1}$ and a parallel coupling line with electrical length $\theta_{2}$, impedance $Z_{2}$. Here $\theta_{c}=\theta_{I O} / 2, \theta_{a}=\theta_{1} / 2-\theta_{c}$ and $\theta_{I O}$ as the length of the electrical input between the input and the output port are defined, and $\theta_{1}+\theta_{2}=\pi$.

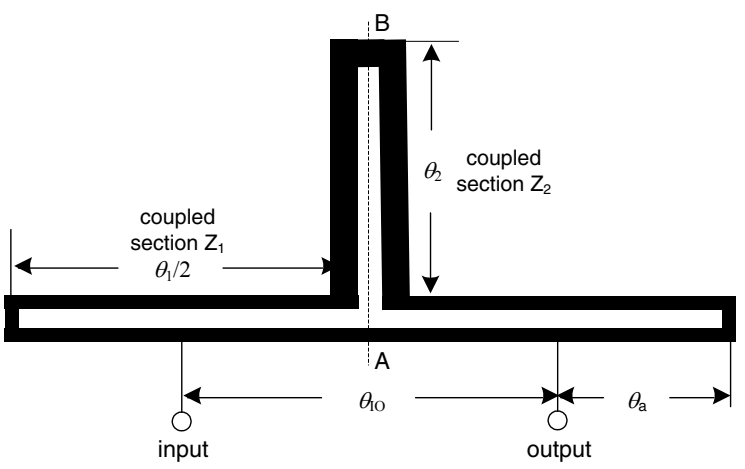

Fig. 6. Self-coupling ring resonator two-way transmission path equivalent circuit.

In Fig. 7, the equations (7) to (12) are the formulas for the parallel coupling microstrip $\mathrm{Y}$ parameter of the four ports and the two ports. Further, the formulas (7) to (12) are converted into the following formulas (13) to (18) using the relationship between the coupling coefficient and the impedance.

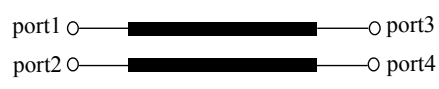

(a)

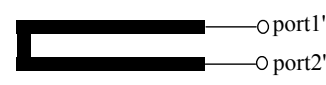

(b)
Fig. 7. parallel coupling transmission line diagram (a) four port (b) two port.

$$
\begin{aligned}
& {\left[\begin{array}{llll}
Y_{11} & Y_{12} & Y_{13} & Y_{14} \\
Y_{21} & Y_{22} & Y_{23} & Y_{24} \\
Y_{31} & Y_{32} & Y_{33} & Y_{34} \\
Y_{41} & Y_{42} & Y_{43} & Y_{44}
\end{array}\right]=\left[\begin{array}{llll}
Y_{11} & Y_{12} & Y_{13} & Y_{14} \\
Y_{12} & Y_{11} & Y_{14} & Y_{13} \\
Y_{13} & Y_{14} & Y_{11} & Y_{12} \\
Y_{14} & Y_{13} & Y_{12} & Y_{11}
\end{array}\right]} \\
& Y_{11}=\left(-\frac{j}{2}\right)\left[Y_{e}+Y_{o}\right] \cot \theta \\
& Y_{12}=\left(-\frac{j}{2}\right)\left[Y_{e}-Y_{o}\right] \cot \theta \\
& Y_{13}=\left(-\frac{j}{2}\right)\left[Y_{e}-Y_{o}\right] \csc \theta \\
& Y_{14}=\left(-\frac{j}{2}\right)\left[Y_{e}+Y_{o}\right] \csc \theta \\
& {\left[\begin{array}{ll}
Y_{11}^{\prime} & Y_{12}^{\prime} \\
Y_{21}^{\prime} & Y_{22}^{\prime}
\end{array}\right]=\left[\begin{array}{ll}
Y_{11}^{\prime} & Y_{12}^{\prime} \\
Y_{12}^{\prime} & Y_{11}^{\prime}
\end{array}\right]} \\
& Y_{11}{ }^{\prime}=\frac{j}{2}\left[Y_{e} \tan \theta-Y_{o} \cot \theta\right] \\
& Y_{12}^{\prime}=\frac{j}{2}\left[Y_{e} \tan \theta+Y_{o} \cot \theta\right] \\
& Y_{11}=Y \frac{-j}{\sqrt{1-c^{2}}} \cot \theta \\
& Y_{12}=Y \frac{-j c}{\sqrt{1-c^{2}}} \cot \theta \\
& Y_{13}=Y \frac{j}{\sqrt{1-c^{2}}} \csc \theta \\
& Y_{14}=Y \frac{-j c}{\sqrt{1-c^{2}}} \csc \theta \\
& Y_{11}{ }^{\prime}=Y \frac{-j}{\sqrt{1-c^{2}}} \cot \theta \\
& Y_{12}{ }^{\prime}=Y \frac{-j}{\sqrt{1-c^{2}} \sin 2 \theta}(c+\cos 2 \theta)
\end{aligned}
$$

By using the formulas (13) to (18), we can solve the position of transmission zero in Fig. 6. The transitive formula is shown in equation (19), and when $Y_{21}$ is zero, the transmission zero is generated.

$$
Y_{21}=Q_{d}+\frac{2 S_{d} T_{d}\left(G_{d}+P_{u}\right)-\left(H_{d}+Q_{u}\right)\left(S_{d}{ }^{2}+T_{d}{ }^{2}\right)}{\left(H_{d}+Q_{u}\right)^{2}-\left(G_{d}+P_{u}\right)^{2}}
$$

And within the formula (19), 


$$
\begin{aligned}
& P_{u}=-\frac{j Y_{2}}{\sqrt{\left(1-c_{2}^{2}\right)} \sin 2 \theta_{2}}\left(c_{2}+\cos 2 \theta_{2}\right), \\
& Q_{u}=\frac{j Y_{2}}{\sqrt{\left(1-c_{2}^{2}\right)} \sin 2 \theta_{2}}\left(1+c_{2} \cos 2 \theta_{2}\right), \\
& S_{d}=Q_{a c}-\frac{S_{a c} G_{a c}}{2 H_{a c}}, T_{d}=-\frac{S_{a c} G_{a c}}{2 H_{a c}}, \\
& G_{d}=T_{a c}-\frac{G_{a c}^{2}}{2 H_{a c}}, H_{d}=-\frac{G_{a c}{ }^{2}}{2 H_{a c}}, Q_{d}=-\frac{Q_{a c}{ }^{2}}{2 H_{a c}}, \\
& Q_{a c}=T_{c}-S_{c} \frac{\left(Q_{a}+Q_{c}\right)}{P_{a}+P_{c}}, \\
& S_{a c}=S_{c}-T_{c} \frac{\left(Q_{a}+Q_{c}\right)}{P_{a}+P_{c}}, \\
& T_{a c}=P_{c}-\frac{S_{c}^{2}}{P_{a}+P_{c}}, \\
& S_{c}=Y_{1} \frac{j}{\sqrt{1-c_{1}^{2}}} \csc \theta_{c}, \quad T_{2}=Y_{1} \frac{-j c_{1}}{\sqrt{1-c_{1}^{2}}} \csc \theta_{c} . \\
& G_{a c}=Q_{c}-\frac{S_{c} T_{c}}{P_{a}+P_{c}}, H_{a c}=P_{c}-\frac{T_{c}^{2}}{P_{a}+P_{c}}, \\
& \cot \theta_{c}, \quad Q_{c}=Y_{1} \frac{j c_{1}}{\sqrt{1-c_{1}^{2}}} \cot \theta_{c},
\end{aligned}
$$

The complicated form of (19) makes it difficult to obtain the analytic solutions in calculation. Then we turn to the graphic expression of $Y_{21}$ for analyzing the effects of the impedance ratio $\mathrm{Z}$ and input-output separation length $\theta_{I O}[9]$.

\subsubsection{Effect of the impedance ratio $Z$}

Fig. 8 is the coupling coefficient with $C_{1}$ and $C_{2}$ being equal to $0.2, \theta_{1}=140^{\circ}, \theta_{2}=40^{\circ}$ and $\mathrm{Z}$ ratio being 0.5 , $1.0,1.8$ in the normalization of the frequency of the $\mathrm{Y}_{21}$ relationship diagram when the angle between the input and output is 80 degree. As shown in the Fig. 8, it can be found that there is no transmission zero on both sides of the fundamental frequency passband when the inductive coupling $Z<1$. When $Z>1$ is capacitively coupled, two transmission zeros are generated on both sides of the passband and a transmission zero occurs near the second passband. When $Z=1$, the transmission zero will occur near the base band, destroying the passband characteristics.

\subsubsection{Effect of angle between input and output ports}

Fig.9 is the normalized frequency of $\mathrm{Y}_{21}$ relationship diagram showing when the coupling coefficient $C_{1}$ and $C_{2}$ are equal to 0.2 , the $\mathrm{Z}$ ratio is 1.8 , with the angle between input port and output being $60^{\circ}, 70^{\circ}, 80^{\circ}, 90^{\circ}$, $100^{\circ}$. It can be seen from the Fig. 9 that when the angle between the input and output port is $70^{\circ} \sim 80^{\circ}$, the passband on both sides of the transmission zero points shows the most symmetry.

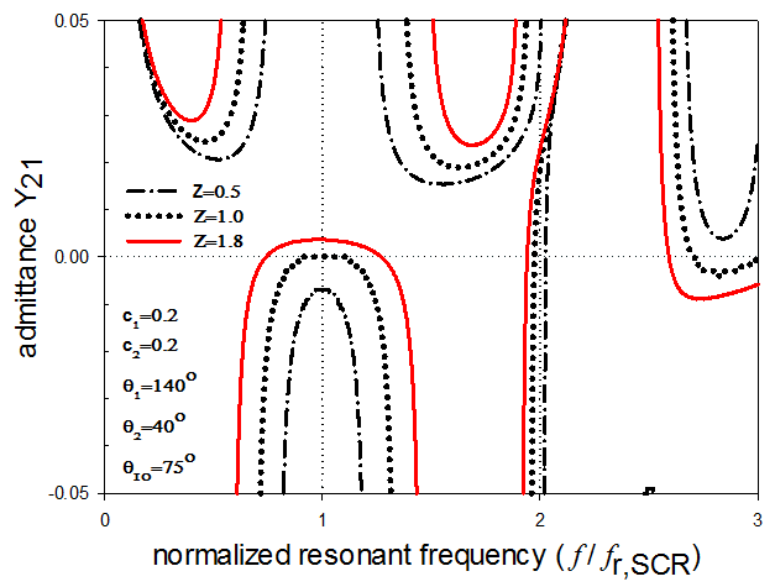

Fig. 8. $\mathrm{Z}$ ratio of the normalization frequency of $\mathrm{Y}_{21}$ relationship diagram.

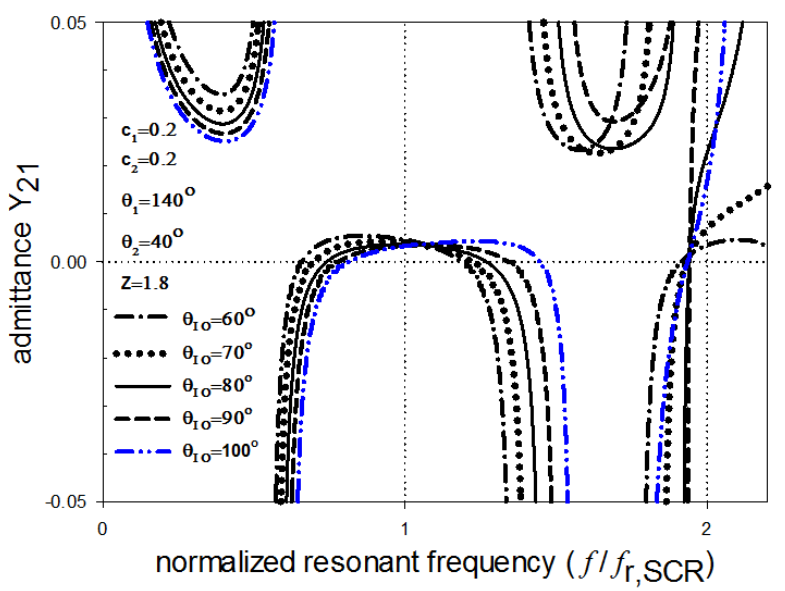

Fig. 9. shows the $Y_{21}$ relationship diagram difference between the input and output ports at the normalized frequency.

\section{Experimential results}

Following the theoretical analysis in section 2, in this paper, the coupling coefficients $C_{1}$ and $C_{2}$ are all used at 0.2 , the electrical length $\theta_{1}=142^{\circ}, \theta_{2}=38^{\circ}$, the characteristic impedance $Z_{1}$ is $109 \Omega, Z_{2}$ is $61.5 \Omega$, and the impedance ratio $Z=1.77$. Moreover, the relative dimensions of the resonator is equivalent to $\mathrm{W}_{2}=1.2$ $\mathrm{mm}, \mathrm{W}_{1}=0.3 \mathrm{~mm}, \mathrm{~W}_{\mathrm{t}}=0.2 \mathrm{~mm}, \mathrm{~L}_{1}=8.5 \mathrm{~mm}, \mathrm{~L}_{2}=4.5 \mathrm{~mm}$, $\mathrm{g}_{2}=0.6 \mathrm{~mm}, \mathrm{~g}_{1}=0.6 \mathrm{~mm}$, and $\mathrm{g}_{\mathrm{t}}=0.2 \mathrm{~mm}$.

This filter is fabricated on the substrate with a relative dielectric constant of 3.38, with loss tangent being 0.0025 and thickness being $0.762 \mathrm{~mm}$. The actual photograph of $5.2 \mathrm{GHz}$ self-coupled bandpass filter is shown in Fig. 10. The size of self-coupled bandpass filter is $9 \times 6 \mathrm{~mm}^{2}$. On the other hand, the circuit size is relatively $54 \%$ of the size to the traditional uniform ring case. 


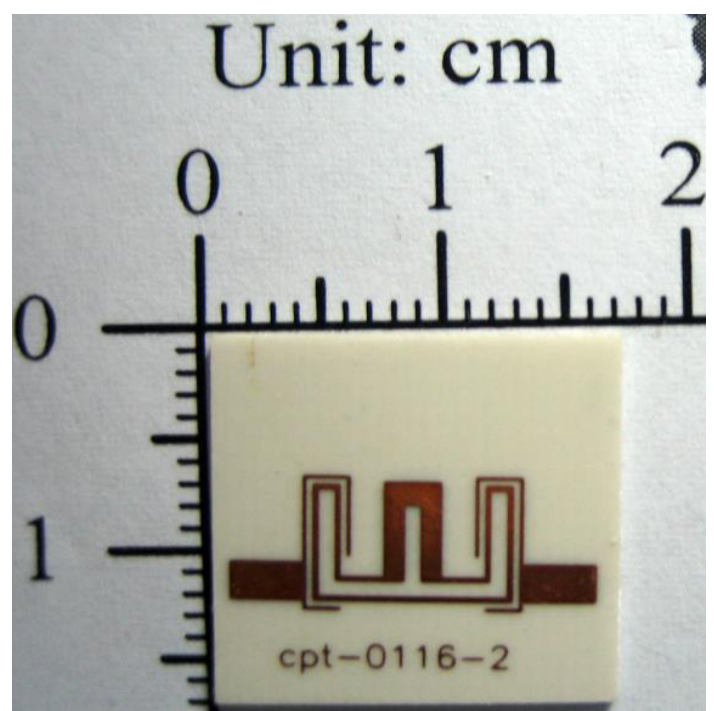

Fig. 10. Photograph of $5.2 \mathrm{GHz}$ bandpass filter.

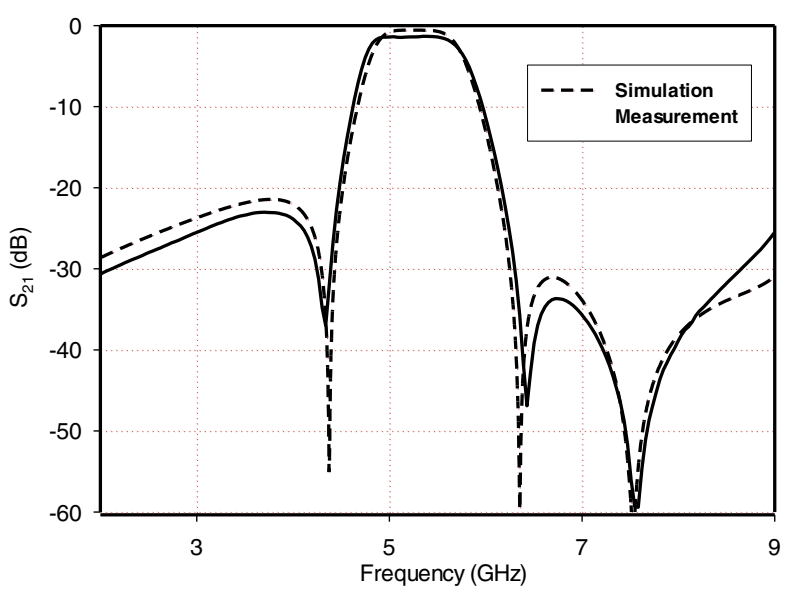

(a) Insertion loss

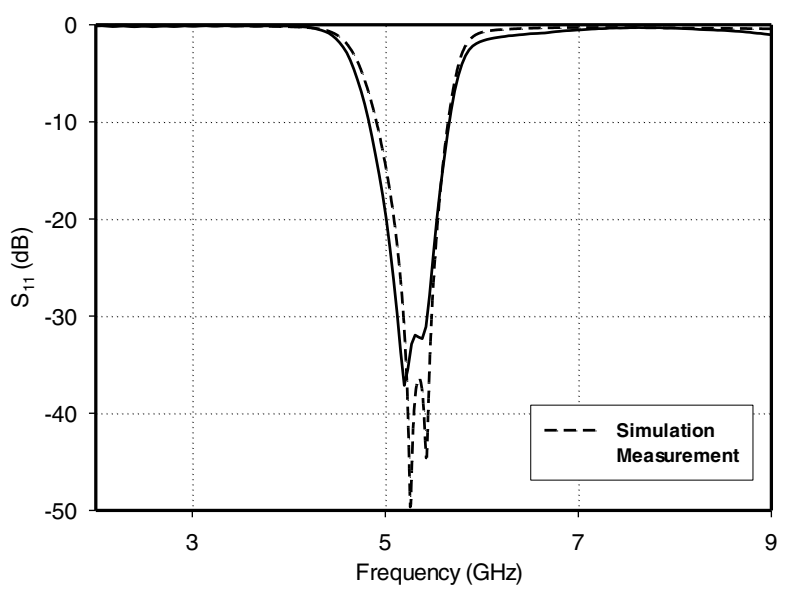

(b) return loss

Fig. 11. shows the measurement and simulation results of bandpass filter.

The simulation and measurement are respectively verified by using IE3D electromagnetic simulator and Agilent's HP8722C network analyzer [10]. Fig. 11 shows the simulated and measured results. Finally, there is very good consensus between the measured and simulated results in this paper that within $5-5.4 \mathrm{GHz}$, the measurement return loss is greater than $22 \mathrm{~dB}$ and measurement insertion loss is less than $1.2 \mathrm{~dB}$ and this condition improves the response of passband. The measured $3-\mathrm{dB}$ bandwidth is about $19.2 \%$ at the center frequency of $5.2 \mathrm{GHz}$. The filter has three transmission zeros close to the passband at $4.39 \mathrm{GHz}, 6.42 \mathrm{GHz}$ and $7.58 \mathrm{GHz}$, which produces sharp bandpass and wide stopband response.

\section{Conclusion}

A $5.2 \mathrm{GHz}$ dual-mode bandpass filter has been proposed in this study by using self-coupled ring resonator for small size, low insertion loss, wide stopband and it is easy to implement. The proposed filter size is $9 \times 6 \mathrm{~mm}^{2}$, three transmission zeros are close to the passband at 4.39 $\mathrm{GHz}, 6.42 \mathrm{GHz}$ and $7.58 \mathrm{GHz}$, and the 3-dB bandwidth is about $19.2 \%$ from $4.75 \mathrm{GHz}$ to $5.73 \mathrm{GHz}$, and insertion loss is less than $1.2 \mathrm{~dB}$ at center frequency 5.2 GHz. A good consensus has been reached in this study by simulation and measurement showing highly valuable application of proposed bandpass filter in wireless communications.

\section{Acknowledgments}

We would like to express our sincere heartfelt thanks to our supervisor, Mr. Sheng-Fuh Chang, professor of Department of Electrical Engineering, National Chung Cheng University, Chiayi, Taiwan, for his invaluable advice and constant help on the paper.

\section{References}

1. I. Wolff, N. Knoppik, Microstrip ring resonator and dispersion measurements on microstrip lines, Electron. Lett., 7 (1971)

2. I. Wolff, Microstrip bandpass filter using degenerate modes of a microstrip ring resonator, Electron. Lett., 8 (1972)

3. M. Matsuo, H. Yabuki, M. Makimoto, Dual-mode stepped-impedance ring resonator for bandpass filter applications, IEEE Trans. Microwave Theory Tech., 49 (2001)

4. S. Luo, L. Zhu, A novel dual-mode dual-band bandpass filter based on a single ring resonator, IEEE Microw. Wireless Compon. Lett., 19 (2009)

5. T.W. Lin, J.T. Kuo, S.J. Chung, Dual-Mode Ring Resonator Bandpass Filter With Asymmetric Inductive Coupling and Its Miniaturization, IEEE Trans. Microwave Theory Tech., 60 (2012)

6. L.H. Hsieh, K. Chang, Compact, low insertion-loss, sharp-rejection, and wide-band microstrip bandpass filters, IEEE Trans. Microwave Theory Tech., 51 (2003) 
7. L. Athukorala, D. Budimir, Compact Dual-Mode Open Loop Microstrip Resonators and Filters, IEEE Microw. Wireless Compon. Lett., 19 (2009)

8. W. Feng, X. Gao, W. Che, Q. Xue, Bandpass Filter Loaded With Open Stubs Using Dual-mode Ring Resonator, IEEE Microw. Wireless Compon. Lett., 25 (2015)

9. Y.H. Jeng, S.F. Chang, Y.M. Chen, Y.J. Hung, A novel self-coupled dual-mode ring resonator and its application to bandpass filters, IEEE Trans. Microwave Theory Tech., 54 (2006)

10. IE3D V10.1, ZELAND SOFTWARE, INC., CA, U.S.A. 